\title{
Case Series about the Changed Antiplatelet Protocol for Carotid Endarterectomy in a Teaching Hospital: More Patients with Complications?
}

Martijn S. Marsman, MD ${ }^{1}$ Denise M.D. Özdemir- van Brunschot, MD ${ }^{2}$ Abdelkarime Kh Jahrome, MD ${ }^{1}$ Nic J.G.M. Veeger, MD, PhD ${ }^{3}$ Wouter J. Schuiling, MD, PhD ${ }^{4}$ Frank G. van Rooij, MD, PhD 4

Giel G. Koning, MD, PhD, FEBVS ${ }^{1}$

\footnotetext{
${ }^{1}$ Department of Vascular Surgery, Medical Center Leeuwarden, Leeuwarden, the Netherlands on behalf of HeelkundeFriesland.nl

2 Department of Vascular Surgery, Augusta Krankenhaus, Düsseldorf, Germany

${ }^{3}$ Department of Epidemiology, MCL Academy, Medical Center

Leeuwarden, Leeuwarden, the Netherlands

${ }^{4}$ Department of Neurology, Medical Center Leeuwarden,

Leeuwarden, the Netherlands
}

Address for correspondence Martijn S. Marsman, MD, Department of Vascular Surgery, Medical Center Leeuwarden, Henri Dunantweg 2, 8935BA Leeuwarden, the Netherlands (e-mail: martijnmarsman@gmail.com).

Surg J 2018;4:e220-e225.

\begin{abstract}
Keywords

- acetylsalicylic acid

- ASA

- bleeding

- carotid endarterectomy

- carotid artery

- clopidogrel

- dipyridamole

- complication

Introduction In the Netherlands, clopidogrel monotherapy increasingly replaces acetylsalicylic acid and extended release dipyridamole as the first-choice antiplatelet therapy after ischemic stroke. It is unknown whether the risk of peri- and postoperative hemorrhage in carotid artery surgery is higher in patients using clopidogrel monotherapy compared with acetylsalicylic acid and extended release dipyridamole. We therefore retrospectively compared occurrence of perioperative major and (clinical relevant) minor bleedings during and after carotid endarterectomy of two groups using different types of platelet aggregation inhibition after changing our daily practice protocol in our center.

Material and Methods A consecutive series of the most recent 80 carotid endarterectomy patients (November 2015-August 2017) treated with the new regime (clopidogrel monotherapy) were compared with the last 80 (January 2012-November 2015) consecutive patients treated according to the old protocol (acetylsalicylic acid and dipyridamole). The primary endpoint was any major bleeding during surgery or in the first 24 to 72 hours postoperatively. Secondary outcomes within 30 days after surgery included minor (re)bleeding postoperative stroke with persistent or transient neurological deficit, persisting or transient neuropraxia, asymptomatic restenosis or occlusion, (transient) headache. Reporting of this study is in line with the 'Strengthening the Reporting of Observational Studies in Epidemiology' statement.

Results Although statistical differences were observed, from a clinical perspective both patients groups were comparable. Postoperative hemorrhage requiring reexploration for hemostasis occurred in none of the 80 patients in the group of the clopidogrel monotherapy (new protocol) and it occurred in one of the 80 patients (1\%) who was using acetylsalicylic acid and dipyridamole (old protocol). In three patients
\end{abstract}

received

July 9,2018

accepted after revision

September 28, 2018
DOI https://doi.org/

$10.1055 / \mathrm{s}-0038-1675566$. ISSN 2378-5128.
Copyright $\odot 2018$ by Thieme Medical

Publishers, Inc., 333 Seventh Avenue, New York, NY 10001, USA Tel: +1(212) 584-4662.
License terms

(1) (1) 
(4\%) in the clopidogrel monotherapy and one patient (1\%) in the acetylsalicylic acid and extended release dipyridamole protocol an ipsilateral stroke was diagnosed.

Conclusion In this retrospective consecutive series the incidence of postoperative ischemic complications and perioperative hemorrhage after carotid endarterectomy (CEA) seemed to be comparable in patients using clopidogrel monotherapy versus acetylsalicylic acid and extended release dipyridamole for secondary prevention after a cerebrovascular event. This study fuels the hypothesis that short- and midterm complications of clopidogrel and the combination acetylsalicylic acid and extended release dipyridamole are comparable.

The neurological and vascular surgical communities established guidelines for antiplatelet therapy as secondary prevention after ischemic stroke or transient ischemic attack (TIA). ${ }^{1}$ The American Heart Association guideline states that the following four antiplatelet regimes are equivalent in the reduction of the risk of ischemic stroke ${ }^{2}$ :

- Acetylsalicylic acid (ASA)

- Clopidogrel

- Ticlopidine

- Combination of ASA and dipyridamole (ASA-D)

Platelet aggregation inhibition is an essential part of secondary prevention after TIA or ischemic stroke. International guidelines approve different antithrombotic regimens for this induction. ${ }^{2,3}$ Based on the ESPRIT trial (Level of Evidence 1d $\left.\left[\mathrm{LoE}^{4}\right]\right)$ the initial antiplatelet regime after TIA or ischemic stroke of presumed arterial origin consisted of ASA $80 \mathrm{mg}$ once a day and extended release dipyridamole $200 \mathrm{mg}$ twice a day. ${ }^{3}$ In the PRoFESS (Prevention Regimen for Effectively Avoiding Second Strokes) trial no differences in cognitive decline or recurrent stroke were found when comparing ASA $25 \mathrm{mg}$ tablets in combination with extended release dipyridamole $200 \mathrm{mg}$ (ASA-D) twice a day versus clopidogrel $75 \mathrm{mg}$ once a day. ${ }^{5}$

With the PRoFESS trial in mind the antithrombotic treatment protocol in the Medical Center Leeuwarden (MCL) was changed from ASA-D to clopidogrel monotherapy in November 2015. Contributory reasons for changing this protocol were the lack of difference in outcome for secondary prevention of a ischemic event between clopidogrel and ASA-D, an increased incidence of headache in patients after starting dipyridamole, a potentially higher compliance for monotherapy, and a cost reduction as clopidogrel monotherapy seems to be less costly then ASA-D. ${ }^{6,7}$ Knowledge about the effectiveness, complications, and costs of clopidogrel monotherapy versus ASA-D may provide insights for other paying parties, such as health insurance companies and hospitals.

Besides lifestyle changes and medication to prevent recurrent TIA or ischemic stroke in the future, patients with a significant and symptomatic carotid artery stenosis require a carotid endarterectomy (CEA). Although antiplatelet medication is important as secondary prevention, it also increases the risk of perioperative hemorrhage. ${ }^{8,9}$ To date, it is unknown whether the risk of perioperative hemorrhage is higher in patients using clopidogrel monotherapy compared with those using ASA-D when undergoing CEA. ASA and clopidogrel monotherapy taken 24 hours before CEA increased post- or perioperative (cervical) bleeding risks. ${ }^{8} 10$ Knowledge of the risk of perioperative hemorrhage in clopidogrel versus ASA-D is important because this can cause a potentially life-threatening situation. In this study, differences in perioperative complications associated with clopidogrel monotherapy versus ASA-D were evaluated in patients who underwent CEA for symptomatic internal carotid artery stenosis. The first aim was to scientifically evaluate outcomes of this safety analysis to provide insight in the complication rate of the operative carotid population of the MCL. These first results on this subject could be used as step up data for a randomized clinical trial.

\section{Material and Methods}

\section{Patients}

Between the change in protocol (November 1, 2015) and the moment of analysis a consecutive series of 80 patients who were treated with clopidogrel monotherapy (November 1 , 2015-August 31, 2017) were compared with the last 80 consecutive patients treated according with ASA-D (January 1 , 2012-November 1, 2015). Antiplatelet therapy was peri- and postoperatively continued in both groups. The perioperative period is defined as the duration between the event (stroke or TIA) after which the antiplatelet therapy started and the moment of the CEA. Patient data were retrieved from the electronic patient files and were analyzed retrospectively. Patients were all invited at the outpatient department for follow up physical examination and carotid ultrasound.

- Inclusion criteria:

- Undergoing unilateral CEA for a symptomatic internal carotid artery stenosis ( $>50 \%$ for the male and $>70 \%$ for the female patients). ${ }^{11}$

- Every patient who underwent a primary CEA in the period from the January 1, 2012 until August 31, 2017 at the MCL.

- Exclusion criteria:

- Use of vitamin K or factor X-a inhibitors.

- Use of other platelet inhibitor combinations.

- Combination of more surgical interventions at the same time. 


\section{Preoperative Procedure}

As part of standard evaluation all patients with TIA or ischemic stroke as diagnosed by an experienced vascular neurologist underwent either computed tomographic angiography or magnetic resonance angiography of the cerebropetal arteries in combination with carotid ultrasonography. When patients were diagnosed with a symptomatic ipsilateral carotid stenosis ( $\geq 50 \%$ in male patients and $\geq 70 \%$ in female patients) they were referred to the vascular surgeon. This is according to the Dutch guidelines that also recommends to perform a CEA within 14 days after the initial ischemic event. ${ }^{11,12}$ Next to these criteria, when the patient is scheduled for CEA in our center, the patient is referred to a dedicated and experienced carotid-team, consisting of a vascular surgeon, neurologist, anesthetist, vascular internist, and a specialized nurse for cardiovascular screening.

\section{Surgical Procedure}

Surgical procedures were unchanged during the study period. Conventional CEA was performed under either general anesthesia or plexus anesthesia. ${ }^{13}$ Conventional CEA was performed, as the carotid eversion technique is not used in MCL given the associated decreased baroreceptor sensitivity which may be related to difficult postoperative tension regulation, and because of the damage that can occur from cutting through the carotid plexus. ${ }^{14,15}$ A 5,000 international units of heparin were given intravenously during CEA. No other hemostatic agents were used. A wound drain was not placed routinely, when placed, it could be removed when it produced less than $25 \mathrm{~mL}$ within 24 hours. The use of a carotid patch angioplasty is not unequivocally proven. ${ }^{16}$ However, in the Dutch vascular surgery guidelines it is recommended. ${ }^{17}$ At the MCL the use of a carotid patch angioplasty depends on the diameter of the carotid artery as can be assessed intraoperatively. All operations were performed by experienced vascular surgeons, all of whom had performed more than 200 CEA procedures. In accordance with our CEA protocol, all patients were closely monitored for the first 24 hours consisting of vital signs, repeated neurological tests and wound inspection. ${ }^{18}$

\section{Endpoints}

The primary endpoint was any bleeding (within 30 days after surgery) requiring hemostasis at the operation room. Secondary outcomes were death within 30 days after surgery, minor bleeding, rebleeding, postoperative ipsi- or contralateral stroke, or TIA, cranial nerve neuropraxia, restenosis or reocclusion, and headache (transient or persisting). Neuropraxia symptoms of cranial nerves were diagnosed by an experienced consultant vascular neurologist.

Endpoints were graded ( - Fig. 1) according to importance for patients according to the GRADE Working Group (Grading of Recommendations Assessment, Development and Evaluation). ${ }^{19}$ This study was conducted and reported in line with the 'Strengthening the Reporting of Observational Studies in Epidemiology' statement (STROBE). ${ }^{20}$

\section{Statistics}

All statistical analyses were performed using SPSS version 24.0 (IBM Corp. Armonk, NY). Values were depicted as mean with standard deviation and number of cases with percentages. Group differences at baseline were assessed using Students $t$-test or Mann-Whitney $U$-test for continuous data (depending on normality of data) and Fisher's exact test or Pearson's Chi-square test for categorical data.

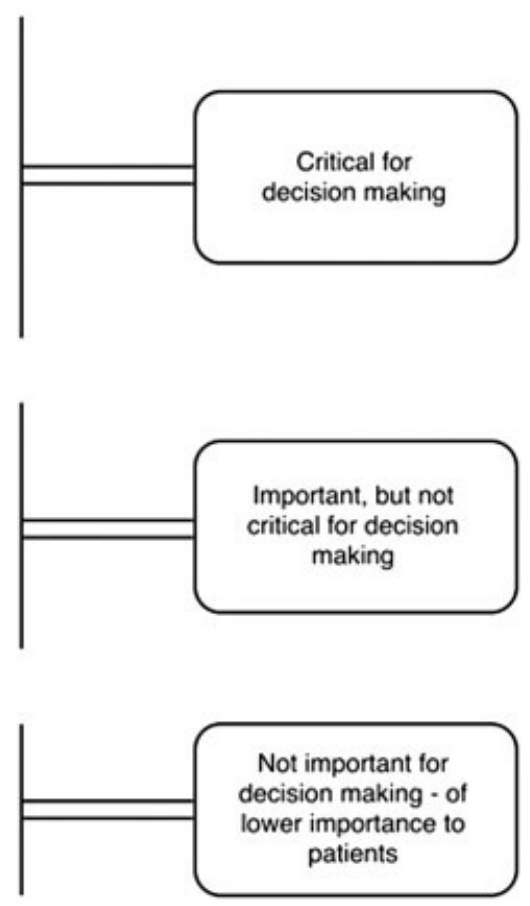

Fig. 1 Example of hierarchy of outcomes according to the GRADE classification, ${ }^{4,19}$ adapted for CEA patients. 
Fisher's exact test was used to test for differences in the occurrence of primary endpoint events. In addition, the exact 95\% confidence intervals (CIs) of point estimates were calculated using the Clopper-Pearson method. For all secondary outcomes, the same analysis approach was used. A p-value of less than 0.05 was used for all tests to indicate statistical significance.

\section{Results}

Between January 2012 and August 2017, a total of 4,514 patients with a stroke or TIA were treated in the MCL. Of these 4,514 patients 252 underwent CEA in line with the indications stated in international carotid guidelines. After exclusion of 92 patients because of predefined criteria, 80 consecutive patients receiving clopidogrel monotherapy were included. In addition, the most recent 80 consecutive patients that used ASA-D were included.

Overall, $73 \%$ of the 160 CEA were performed within 14 days after the initial ischemic event. In cases where the time frame of treatment was not attained, the reason for this was patient delay or prehospital referral delay. Patient demographics are shown in - Table 1 and perioperative parameters are presented in - Table 2.

Although significant differences were found in - Tables 1 and $\mathbf{2}$, these differences were considered to be not clinically relevant.

Although significant differences were found in - Table $\mathbf{2}$, these differences were considered not to be clinically relevant.

Complications are shown in -Table 3. Postoperative hemorrhage requiring return to the operation room occurred in no patients in the clopidogrel monotherapy protocol and in one $(1 \%)$ in the ASA-D protocol. Three patients using clopidogrel and one patient using ASA-D developed an ipsilateral ischemic stroke. In the 30 day follow-up period
Table 2 Perioperative parameters

\begin{tabular}{|l|l|l|l|}
\hline & $\begin{array}{l}\text { Clopidogrel } \\
(\boldsymbol{n}=\mathbf{8 0})\end{array}$ & $\begin{array}{l}\text { ASA-D } \\
(\boldsymbol{n}=\mathbf{8 0})\end{array}$ & $p$-Value \\
\hline Right sided CEA & $34(43 \%)$ & $35(44 \%)$ & 1.00 \\
\hline $\begin{array}{l}\text { Median delay from } \\
\text { event to surgery } \\
\text { in d (IQR) }\end{array}$ & $11(8-14)$ & $11(9-14)$ & 0.96 \\
\hline Patch used & $39(49)$ & $70(88)$ & $<0.001$ \\
\hline Dacron & $2(5)$ & $1(1)$ & \\
\hline Biopatch & $36(92)$ & $67(96)$ & \\
\hline Venous & $1(3)$ & $2(3)$ & $18(23)$ \\
\hline Shunting needed & $20(25)$ & 0.85 \\
\hline $\begin{array}{l}\text { Median duration } \\
\text { of surgery in } \\
\text { min (IQR) }\end{array}$ & $\begin{array}{l}90 \\
(75-100)\end{array}$ & $\begin{array}{l}90 \\
(90-100)\end{array}$ & 0.08 \\
\hline Anesthesia & $78(98)$ & $80(100)$ & \\
\hline General & $2(2)$ & $0(0)$ & \\
\hline Plexus & & & \\
\hline
\end{tabular}

Abbreviations: ASA-D, acetylsalicylic acid and extended release dipyridamole; Biopatch, bovine; IQR, Interquartile Range.

Note: Data are presented as $n(\%)$ unless stated otherwise.

two patients died, both in the clopidogrel group. One patient died because of a postoperative ipsilateral stroke and one due to an aspiration pneumonia. The CT-scan (computed tomography scan) of the patient with the stroke showed no recent ischemia or bleeding in the brain. There was, in contrast to a CT prior to the CEA, no significant stenosis of the internal carotid artery. No acute stops or thrombosis in the artery were seen. The aspiration pneumonia was seen on X-ray, there was not a CT scan of the brain made - Table 3.

Table 1 Patients demographics and surgical parameters, stratified by antiplatelet therapy regime

\begin{tabular}{|l|l|l|l|}
\hline & Clopidogrel $(\boldsymbol{n}=\mathbf{8 0})$ & ASA-D $(\boldsymbol{n}=\mathbf{8 0})$ & $p$-Value \\
\hline Period & November 2015-August 2017 & January 2012-November 2015 & \\
\hline Mean age in y (SD) & $73(9.8)$ & $71(10.8)$ & 0.19 \\
\hline Male & $46(58)$ & $55(69)$ & 0.19 \\
\hline Comorbidity ${ }^{a}$ & $59(74)$ & $72(90)$ & 0.01 \\
\hline Hypertension & $45(47)$ & $58(42)$ & \\
\hline Diabetes & $20(21)$ & $23(17)$ & \\
\hline Hypercholesterolemia & $31(32)$ & $57(41)$ & \\
\hline Smoking & $53(66)$ & $49(61)$ & 0.49 \\
\hline Presenting event & & & 0.87 \\
\hline TIA & $36(45)$ & $38(47)$ & \\
\hline Ischemic stroke & $44(55)$ & $42(53)$ & \\
\hline $\begin{array}{l}\text { Recurrent events between } \\
\text { first presentation and CEA }\end{array}$ & $0(0)$ & $0(0)$ & NC \\
\hline
\end{tabular}

Abbreviations: ASA-D, acetylsalicylic acid and extended release dipyridamole; CEA, carotid endarterectomy; NC, not computable; SD, standard deviation; TIA, transient ischemic attack.

Note: Data are presented as $n(\%)$ unless stated otherwise.

ane or more. 
Table 3 Overview of complications in CEA patients ${ }^{19,21}$

\begin{tabular}{|l|l|l|l|l|l|}
\hline & \multicolumn{2}{|l|}{ Clopidogrel $(\boldsymbol{n}=\mathbf{8 0})$} & \multicolumn{2}{l|}{ ASA-D $(\boldsymbol{n}=\mathbf{8 0})$} & $p$-Value \\
\hline Cervical hemorrhage $^{\mathrm{a}}$ & $0(0)$ & $(95 \% \mathrm{Cl} 0.0-4.5)$ & $1(1.2)$ & $(95 \% \mathrm{Cl} 0.03-6.7)$ & 1.0 \\
\hline Ischemic stroke: ipsilateral & $2(2.5)$ & $(95 \% \mathrm{Cl} 0.7-8.6)$ & $1(1.2)$ & $(95 \% \mathrm{Cl} 0.03-6.7)$ & 1.0 \\
\hline Ischemic stroke: contralateral & $0(0)$ & $(95 \% \mathrm{Cl} 0.0-4.5)$ & $0(0)$ & $(95 \% \mathrm{Cl} 0.0-4.5)$ & $\mathrm{NC}$ \\
\hline Mortality $(\leq 30 \mathrm{~d})$ & $2(2.5)$ & $(95 \% \mathrm{Cl} 0.7-8.6)$ & $0(0)$ & $(95 \% \mathrm{Cl} 0.0-4.5)$ & 0.50 \\
\hline Neuropraxia of cranial nerves & $7(8.8)$ & $(95 \% \mathrm{Cl} 3.6-17.2)$ & $9(11)$ & $(95 \% \mathrm{Cl} 5.3-20.3)$ & 0.86 \\
\hline Persisting $(\geq 30 \mathrm{~d})$ & $3(42.8)$ & & $4(44.4)$ & & \\
\hline Transient $(<30 \mathrm{~d})$ & $4(57.2)$ & & $5(55.5)$ & & \\
\hline Headache and/or hypertension & $51(63.8)$ & $(95 \% \mathrm{Cl} 52.2-74.2)$ & $61(76.3)$ & $(95 \% \mathrm{Cl} 65.4-85.1)$ & 0.12 \\
\hline Persisting headache $(\geq 30 \mathrm{~d})$ & $1(12.5)$ & & $0(0)$ & & \\
\hline
\end{tabular}

Abbreviations: $95 \% \mathrm{Cl}, 95 \%$ confidence interval; ASA-D, acetylsalicylic acid and extended release dipyridamole; NC, not computable.

Note: Data are presented as $n(\%)$ unless stated otherwise.

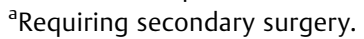

\section{Discussion}

In this retrospective analysis no differences were found in periand postoperative stroke and hemorrhage after CEA between patients using clopidogrel monotherapy and ASA-D. There were no differences found in the other defined outcomes in both groups (clopidogrel monotherapy and ASA-D).

The limitation of this study is its retrospective nature which could imply inaccurately recording of some peri- and postoperative clinical parameters, e.g., hematoma. Therefore we interpret the results cautiously. We use these data as step up study for further research. Also the case-mix, treatment and outcome are impressible to a certain risk of bias. The incidence of cervical bleeding/hematoma after CEA is not easy to observe reliably and there could have been substantial difference in its definition. Nevertheless major bleeding, in need of a reoperation were well documented and therefore considered as an objective count. Postoperative there are clear symptoms for the nurse and doctor to check. If there was any suspicion of a stroke a neurologist was consulted. Therefore the primary endpoints can be assumed to be well documented.

There is a significant difference between the clopidogrel monotherapy and the ASA-D groups regarding the use of a patch. This may be true or could contain a risk for bias. A patch is used depending on the diameter of the carotid artery, a variable that was not reported in all operation reports. Some studies previously compared the effect of antiplatelet therapy as secondary prevention after stroke. ${ }^{22-24}$ Studies specifically comparing the effect of antiplatelet therapy prior to and shortly after CEA are scarce. In the study by Stone et al (2011), reoperation for bleeding was significantly higher in the ASA/ clopidogrel group (1.4\%) versus ASA monotherapy (1.2\%) or clopidogrel monotherapy (0.7\%) after CEA(LoE 2b). ${ }^{7}$ In the study by Weinrich et al (2014), the incidence of revision surgery was also higher for patients on ASA in combination with any other antiplatelet drug (commonly clopidogrel) when compared with ASA monotherapy (LoE 2b). ${ }^{25}$ In both studies the incidence of postoperative CVA or TIA's are not mentioned. Other studies confirmed these findings. ${ }^{26,27}$ When comparing ASA-D dual therapy to triple therapy (dipyridamole, ASA, and clopidogrel) no significant differences in postoperative microembolic signals or bleeding complications were demonstrated. ${ }^{28}$

Literature suggests the costs of clopidogrel are lower than ASA-D. The costs of clopidogrel monotherapy in the Netherlands vary between $€ 1.18$ to 11.83 per patient/per 30 days depending on the manufacturer (with a maximum of $€ 143.98$ annually). The combination ASA-D costs at minimum $€ 15.24$ and $€ 16.13$ per patient/per 30 days at maximum (and a maximum of $€ 196.29$ annually). ${ }^{29}$

The results of this study are in line with previously published literature. ${ }^{30}$ The changed antiplatelet protocol did not result in more complications in our consecutive series. Nevertheless more clinical research may be needed to confirm or reject these data before firm conclusions can be drawn. It can be hypothesized that clopidogrel monotherapy could contribute to general therapy compliance and seems to be more cost effective on annual basis for the CEA patients in our investigated series. It may be suggested that clopidogrel should be the preferred choice for CEA patients.

\section{Conclusion}

In this retrospective consecutive series the incidence of postoperative ischemic complications and perioperative hemorrhage after CEA seemed to be comparable in patients using clopidogrel monotherapy versus acetylsalicylic acid and extended release dipyridamole for secondary prevention after a cerebrovascular event. This study fuels the hypothesis that short- and midterm complications of clopidogrel and the combination acetylsalicylic acid and extended release dipyridamole are comparable.

\section{Funding}

This research did not receive any specific grant form funding agencies in the public, commercial or not-for-profit sectors.

\section{Presentation}

This study was presented at the annual meeting of the Dutch Society of Vascular Surgery (NVvV) on November $2^{\text {nd }} 2018$. 


\section{Conflict of Interest}

None.

\section{References}

1 Kwok CS, Shoamanesh A, Copley HC, Myint PK, Loke YK, Benavente OR. Efficacy of antiplatelet therapy in secondary prevention following lacunar stroke: pooled analysis of randomized trials. Stroke 2015;46(04):1014-1023

2 Furie KL, Kasner SE, Adams RJ, et al; American Heart Association Stroke Council, Council on Cardiovascular Nursing, Council on Clinical Cardiology, and Interdisciplinary Council on Quality of Care and Outcomes Research. Guidelines for the prevention of stroke in patients with stroke or transient ischemic attack: a guideline for healthcare professionals from the american heart association/american stroke association. Stroke 2011;42(01):227-276

3 Halkes PH, van Gijn J, Kappelle LJ, Koudstaal PJ, Algra A; ESPRIT Study Group. Aspirin plus dipyridamole versus aspirin alone after cerebral ischaemia of arterial origin (ESPRIT): randomised controlled trial. Lancet 2006;367(9523):1665-1673

4 Keus F, Wetterslev J, Gluud C, van Laarhoven CJ. Evidence at a glance: error matrix approach for overviewing available evidence. BMC Med Res Methodol 2010;10:90

5 Diener HC, Sacco RL, Yusuf S, et al; Prevention Regimen for Effectively Avoiding Second Strokes (PRoFESS) study group. Effects of aspirin plus extended-release dipyridamole versus clopidogrel and telmisartan on disability and cognitive function after recurrent stroke in patients with ischaemic stroke in the Prevention Regimen for Effectively Avoiding Second Strokes (PRoFESS) trial: a double-blind, active and placebo-controlled study. Lancet Neurol 2008;7(10):875-884

6 Brown MT, Bussell JK. Medication adherence: WHO cares? Mayo Clin Proc 2011;86(04):304-314

7 Stone DH, Goodney PP, Schanzer A, et al; Vascular Study Group of New England. Clopidogrel is not associated with major bleeding complications during peripheral arterial surgery. J Vasc Surg 2011;54(03):779-784

8 Baracchini C, Gruppo M, Mazzalai F, Lorenzetti R, Meneghetti G, Ballotta E. Predictors of neck bleeding after eversion carotid endarterectomy. J Vasc Surg 2011;54(03):699-705

9 Wait SD, Abla AA, Killory BD, Starke RM, Spetzler RF, Nakaji P. Safety of carotid endarterectomy while on clopidogrel (Plavix). Clinical article. J Neurosurg 2010;113(04):908-912

10 Morales Gisbert SM, Sala Almonacil VA, Zaragozá García JM, Genovés Gascó B, Gómez Palonés FJ, Ortiz Monzón E. Predictors of cervical bleeding after carotid endarterectomy. Ann Vasc Surg 2014;28(02):366-374

11 Rothwell PM, Eliasziw M, Gutnikov SA, Warlow CP, Barnett HJ; Carotid Endarterectomy Trialists Collaboration. Endarterectomy for symptomatic carotid stenosis in relation to clinical subgroups and timing of surgery. Lancet 2004;363(9413):915-924

12 Neurologie NVvN. Diagnostiek, behandeling en zorg voor patiënten met een beroerte. 2008 Guideline. Available from: https://internisten.nl/sites/internisten.nl/files/uploads/6N/EE/ 6NEEZDkyKDcbjVkcazdTMQ/richtlijn_2008_Beroerte.pdf. Accessed October 24, 2017

13 Rerkasem K, Rothwell PM. Local versus general anaesthesia for carotid endarterectomy. Cochrane Database Syst Rev 2008;(04): CD000126

14 Demirel S, Macek L, Bruijnen H, Hakimi M, Böckler D, Attigah N. Eversion carotid endarterectomy is associated with decreased baroreceptor sensitivity compared to the conventional technique. Eur J Vasc Endovasc Surg 2012;44(01):1-8

15 Koning GG, Lüning TH, ter Bruggen JP. Een vrouw met linkszijdig retrobulbaire pijn, fotofobie en hornersyndroom. Nederlands Tijdschrift voor Geneeskunde (Stud. ed.). 2007;10(3):55-57

16 Karimi A, van der Vliet JA, Wetterslev J, et al. Protocol: primary closure versus patch angioplasty in carotid endarterectomy: a systematic review with meta-analyses and trial sequential analyses of randomized clinical trials 2014. Available from: http://www.crd. york.ac.uk/PROSPEROFILES/13416_PROTOCOL_20140726.pdf. Accessed October 16, 2018

17 Markus HS, Droste DW, Kaps M, et al. Dual antiplatelet therapy with clopidogrel and aspirin in symptomatic carotid stenosis evaluated using doppler embolic signal detection: the Clopidogrel and Aspirin for Reduction of Emboli in Symptomatic Carotid Stenosis (CARESS) trial. Circulation 2005;111(17):2233-2240

18 Doig D, Turner EL, Dobson J, et al; ICSS Investigators. Risk factors for stroke, myocardial infarction, or death following carotid endarterectomy: results from the international carotid stenting study. Eur J Vasc Endovasc Surg 2015;50(06):688-694

19 Guyatt GH, Oxman AD, Kunz R, et al. GRADE guidelines 6. Rating the quality of evidence-imprecision. J Clin Epidemiol 2011;64 (12):1283-1293

20 von Elm E, Altman DG, Egger M, Pocock SJ, Gøtzsche PC, Vandenbroucke JP; STROBE Initiative. The Strengthening the Reporting of Observational Studies in Epidemiology (STROBE) statement: guidelines for reporting observational studies. J Clin Epidemiol 2008;61(04):344-349

21 Guyatt GH, Oxman AD, Kunz R, Vist GE, Falck-Ytter Y, Schünemann HJ; GRADE Working Group. What is "quality of evidence" and why is it important to clinicians? BMJ 2008;336(7651):995-998

22 Davis KA, Miyares MA, Dietrich E. Dual antiplatelet therapy with clopidogrel and aspirin after ischemic stroke: a review of the evidence. Am J Health Syst Pharm 2015;72(19):1623-1629

23 Brandler ES, Sharma M. Early dual antiplatelet therapy in stroke: should we take the CHANCE? Ann Transl Med 2015;3(13):177

24 Tan S, Xiao X, Ma H, et al. Clopidogrel and aspirin versus aspirin alone for stroke prevention: a meta-analysis. PLoS One 2015;10 (08):e0135372

25 Weinrich M, Schindler P, Kundt G, Klar E, Bünger CM. Influence of local hemostatic and antiplatelet agents on the incidence of bleeding complications in carotid endarterectomies. Clin Hemorheol Microcirc 2014;58(01):271-279

26 Hale B, Pan W, Misselbeck TS, Lee VV, Livesay JJ. Combined clopidogrel and aspirin therapy in patients undergoing carotid endarterectomy is associated with an increased risk of postoperative bleeding. Vascular 2013;21(04):197-204

27 Oldag A, Schreiber S, Schreiber S, et al. Risk of wound hematoma at carotid endarterectomy under dual antiplatelet therapy. Langenbecks Arch Surg 2012;397(08):1275-1282

28 de Borst GJ, Hilgevoord AA, de Vries JP, et al. Influence of antiplatelet therapy on cerebral micro-emboli after carotid endarterectomy using postoperative transcranial Doppler monitoring. Eur J Vasc Endovasc Surg 2007;34(02):135-142

29 Medicijnkosten.nl. [Internet]. www.medicijnkosten.nl. Zorginstituut Nederland; 2017. Available from: https://www.medicijnkosten.nl/databank? medicijnId=1697\&hoeveelheid=1\&niveau=2. Accessed November 24, 2017

30 Illuminati G, Schneider F, Pizzardi G, Masci F, Calio' FG, Ricco JB. Dual antiplatelet therapy does not increase the risk of bleeding after carotid endarterectomy: results of a prospective study. Ann Vasc Surg 2017;40:39-43 